\title{
Maternal Serum Triple Screening Test and Pregnancy Outcome in Elderly Sudanese Pregnant Ladies in Khartoum State-Sudan
}

\author{
Rimaz A. Gurashi ${ }^{1}$, Imad Fadl-Elmula ${ }^{2}$ \\ ${ }^{1}$ Clinical Chemistry Department, Faculty of Medical Laboratory Sciences, Al-Neelain University, Khartoum, Sudan \\ ${ }^{2}$ Safa Academy, Khartoum, Sudan
}

Email address:

rimazgourashi@gmail.com (R. A. Gurashi),imad@fadl-elmula.com (I. Fadl-Elmula)

\section{To cite this article:}

Rimaz A. Gurashi, Imad Fadl-Elmula. Maternal Serum Triple Screening Test and Pregnancy Outcome in Elderly Sudanese Pregnant Ladies in Khartoum State-Sudan. American Journal of Laboratory Medicine. Vol. 2, No. 6, 2017, pp. 172-178. doi: 10.11648/j.ajlm.20170206.19

Received: January 29, 2017; Accepted: March 10, 2017; Published: November 28, 2017

\begin{abstract}
The possibility to deliver infants with congenital defects or diseases is thought to be associated with increased in maternal age. This fact is well established and documented in previous studies done in the United States of America. Infants with birth defects is not the only disastrous outcome, pregnancy and delivery complications, abortion, stillbirth are also known consequences. The aim of this study, to detect a possible association between increased maternal age, and Triple Screening Test results. And compare the findings of the Triple Screening Test results with abnormal pregnancy outcome among Sudanese pregnant ladies. Study performed in ninety-one pregnant ladies accepted to participate. Participants were assigned to two groups; study group including $69(75.8 \%)$ women equal to and above 35 years and control group including $22(24.2 \%)$ women less than 30 years. Structured questionnaire, clinical examinations including ultra-sound for the determination of gestational age were also performed. Blood samples were collected from all participants during the $14^{\text {th }}$ to $20^{\text {th }}$ week of gestational age. Sera were separated and used for the determination of human chorionic gonadotropin, alpha fetoprotein, and free estriol concentrations using standardized quantitative methods (ELISA) collectively known as the Triple Screening Test. In the study group, participants delivered $57(82.6 \%)$ normal healthy infants. Twelve ladies $(17.4 \%)$ delivered infants with adverse dimorphic features. The defects varied; four $(5.8 \%)$ had stillbirth, four $(5.8 \%)$ had abortion at the second trimester, three (4.3\%) had premature delivery, and one (1.4\%) had intrauterine death. And matching control group, none of the participants $(0 \%)$ give birth to an infant with congenital defect. Highly significant positive correlation was detected between increase in maternal age and abnormal pregnancy outcome. The Triple Screening Test against the pregnancy outcome in study group, showed statistically significant differences between levels of $\beta$-HCG, AFP, and Free estriol and pregnancy outcomes with Pvalues of $0.000,0.5240$, and 0.000 were obtained using Pearson Chi-squire test of significance, respectively. Study conclude that abnormal pregnancy outcome is strongly associated with increase in maternal age. Also there were strongly association between the Triple Screening Test and abnormal pregnancy outcome.
\end{abstract}

Keywords: Maternal, Pregnancy Outcome, Elderly, HCG, AFP, Free Estriol, ELISA

\section{Introduction and Literature Review}

Pregnancy is the carrying of one or more offspring, known as a fetus or embryo, inside the uterus of a human female. In a pregnancy, there can be multiple gestations, as in the case of twins or triplets, occurs as the result of the female egg being penetrated by the male spermatozoon in a process of fertilization, or more commonly known as "conception" [16].

Though pregnancy begins at implantation, it is more convenient to date from the first day of a woman's last menstrual period (LMP), or from the date of conception. Starting from one of these dates, the expected date of delivery (EDD) can be calculated, counting from the LMP (Naegele's rule for estimating date of delivery). Normal pregnancy lasts approximately 40 weeks as measured from the first day of the last normal menstrual period; in pregnancy a woman undergoes dramatic physiological and hormonal changes. 
Pregnancy is divided into three time intervals called trimesters, each of which is slightly longer than 13 weeks. Each trimester of pregnancy is marked by developmental changes in the fetus [27].

Pregnancy Diagnosis, the beginning of pregnancy may be detected in a number of ways, including various pregnancy tests, which detect hormones generated by the newly formed placenta. Clinical blood and urine tests can detect pregnancy soon after implantation, which is as early as 6-8 days after fertilization. Home pregnancy tests (qualitative test) are personal urine tests, which normally cannot detect a pregnancy until at least 12-15 days after fertilization. Both clinical and home tests can only detect the state of pregnancy, and cannot detect its age. An early sonograph can determine the age of the pregnancy accurately. In practice, doctors typically express the age of a pregnancy (i.e. an "age" for an embryo) in terms of "menstrual date" based on the first day of a woman's last menstrual period [29] as the woman reports it [9]. Unless a woman's has been charting her cycles, or the conception is as the result of some types of fertility treatment such as intrauterine insemination IUI or in-vitro fertilization IVF the exact date of fertilization is unknown. The expected date of delivery may also be calculated from sonogram measurement of the fetus. This method is slightly more accurate than methods based on LMP [29]. Finally, the diagnostic criteria are Women who have menstrual cycles and are sexually active, a period delayed by a few days or weeks is suggestive of pregnancy; and elevated $\beta$-hcg to around $100,000 \mathrm{mIU} / \mathrm{mL}$ by 10 weeks of gestation [5].

Pregnancy in elderly, as women reach their thirties, they experience a decline in fertility. Age-related decline in fertility may be due, in part, to the following: A decrease in the number and health of the eggs to be ovulated, a decrease in the frequency of intercourse, The presence of other medical and gynecologic conditions, such as endometriosis, which may interfere with conception health complications for this age group (at age 40 years and older) are higher than for younger women [32].

If they have decided to delay having a child, they should understand the risks associated with this, so they can take precautions to minimize risks and improve there chances for a healthy pregnancy and child. The risk of giving birth to a child with a birth defect does increase as the mother's age increases. This is probably due to abnormal division of the egg, called no disjunction. The traditional age at which a woman is considered to be at high risk for chromosomal abnormalities is 40 and also miscarrying which is the loss of pregnancy before 20 weeks gestation [14].

Birth defects, are defined as abnormalities of structure, function, or body metabolism that are present at birth. These abnormalities lead to mental or physical disabilities or are fatal. There are more than 4,000 different types of birth defects ranging from minor to serious, and although many of them can be treated or cured, they are the leading cause of death in the first year of life. According to the March of Dimes, about 150,000 babies are born with birth defects each year in the United States. The American College of
Obstetricians and Gynecologists (ACOG) says that out of every 100 babies born in the United States, three have some kind of major birth defect. Birth defects can be caused by genetic, environmental, or unknown factors.

Causes of Birth Defects, most babies with birth defects are born to two parents with no obvious health problems or risk factors. In fact, about $60 \%$ of birth defects have unknown causes. The rest are caused by genetic or environmental factors, or some combination of the two. Many birth defects are severe or even fatal, but the fact is that many are treatable, often immediately after birth and sometimes even before the baby is born. It is especially important to know the risk factors involved and what we can do to prevent birth defects. However, it is also important to realize that most children born with congenital defects are born to two healthy parents.

Common causes are genetic causes, environmental causes, and multifactorial causes,

Other causes, include $\mathrm{Rh}$ disease, which can occur when the mother's and baby's Rh factors are different. Although a few medications can cause problems, of the 200 most commonly prescribed drugs, none is associated with a significant risk of birth defects.

Detections of birth defects, There are varieties of techniques available for prenatal diagnosis; each of them can be applied only during specific periods during the pregnancy for greatest utility. The techniques employed for prenatal diagnosis classified into screening and diagnostic tests or invasive and noninvasive techniques. The invasive techniques Include Amniocentesis, Chorionic villus sampling, fetal blood cells in maternal blood (diagnostic test) noninvasive are Triple screen and ultrasound (screening test) [18].

Amniocentesis, is a diagnostic technique, which performed between 14 and 20 weeks gestation. A procedure in which a small amount amniotic fluid is removed from the sac surrounding the fetus and tested for birth defects by estimation of AFP in amniotic fluid. Risks with amniocentesis are fetal mortality about $0.5 \%$ and maternal $\mathrm{Rh}$ sensitization [2].

Chorionic villus sampling, is sampling of cells from the placental chorionic villi by using a catheter, which passed via the vagina through the cervix and into the uterus to the developing placenta under ultrasound guidance, the risks of this procedure are loss rate is about 0.5 to $1 \%$. Associated with limb defects in the fetus. The possibility of maternal $\mathrm{Rh}$ sensitization is present [2].

Fetal blood cells in maternal blood, is a new technique, use of the phenomenon of fetal blood cells gaining access to maternal circulation through the placental villi, in fetal cells looks for DNA sequence, Fluorescence in-situ hybridization (FISH) is one technique that can be applied, but it is difficult to get many fetal blood cells [2].

Non-invasive techniques, are (screening tests) which is harmless for fetus and mother, common screening test included ultrasound and triple screening test [11].

Ultrasonography, define as medical sonography 
(ultrasonography) is an ultrasound-based diagnostic imaging technique used to visualize muscles and internal organs, their size, structures and possible pathologies or lesions. Obstetric sonography is commonly used during pregnancy used to monitor the health of pregnancy and the growing fetus, which called obstetrical ultrasound [5]. An estimate of gestational age by ultrasound examination improves the performance of the triple test., the use of ultrasound was found to raise the sensitivity of the triple test from 60 percent to 74 percent and to decrease the initial false-positive rate from 9 percent to 5 percent. When available, an ultrasound estimate of gestational age should be provided to the laboratory instead of the due date based on the patient's last menstrual period (Kasai C. et al., 1985). The purpose of this scan should be to establish the gestational age accurately, viability, fetal number, multiple pregnancies, the chorionicity/amnionicity and, detection of gross fetal abnormalities [32].

Triple screening markers, (also called the maternal serum screening test or multiple marker test), is a blood test that is performed usually between the 14th and 20th week of pregnancy. This screening test measures the levels of three substances, alpha-fetoprotein (AFP), human chrionic gonadotropin (hCG), and unconjugated estriol (uE3) in the maternal blood [20].

It is very important that the correct gestational age be determined by last menstrual period dating LMP and recorded for the risk calculation. Errors in determining the age of the fetus lead to errors when interpreting the test results. Since the triple maternal serum test is only a screening tool, an abnormal test result is not necessarily indicative of a birth defect. Accurate gestational dating lowers the false-positive and false-negative rates associated with this screening test [20] [16].

Alpha Fetoprotein (AFP) is an oncofetal antigen having 70 KD molecular weight glycoprotein in nature, it consist of a single polypeptide chain and is $4 \%$ carbohydrate. Which synthesized early in fetal development by the yolk sac, and later by the liver. AFP is related closely both genetically and structurally to albumin, having extensive homologies in amino acids sequence. The gene coding for both protein have been localized to chromosome 4q. AFP being replaced as the predominated protein in serum by albumin. They finally reach the trace concentration found in normal adults 18 months after birth.

During pregnancy, maternal AFP level increase from 12 weeks of gestation to a peak of about $300 \mu \mathrm{g} / \mathrm{l}$ during the third trimester the fetal AFP reaches a peak of $2 \mathrm{~g} / 1$ in 14 weeks of gestation and then declines about $70 \mathrm{mg} / \mathrm{l}$ at term., AFP leaks across the placenta into the maternal circulation during pregnancy [37], the amount depending on the amount produced by the fetus, the increasing of MS-AFP is correlated with types of birth defect.

The clinical implication of MS-AFP are elevated in cases of neural tube defects, underestimation of gestational age, threatened spontaneous abortion, and as increased placental transfer also occur in multiple pregnancies and low MS-AFP is associated with log standing fetal death, down syndrome, tiresome 13, tiresome 18, and pseudo pregnancy [14]. Interfering factors, obesity cause low MS-AFP, race (higher in black from $10 \%$ to $15 \%$ low in Asian and also insulin dependent diabetes mellitus results in low MS-AFP.

Human chrionic gonadotropin (hcg) is a glycoprotein hormone with two nonidentical subunits (alpha and beta polypeptide chains). It has structural similarities to other hormones such as luteinizing hormone ( $\mathrm{LH})$ and follicle stimulating hormone (FSH), particularly in the alpha chain, while the beta chain is unique to HCG. This hormone is produced by chorionic villi of the implanted blastocyst and triggers the corpus luteum to release progesterone and estrogen. HCG helps maintain the uterine lining, the endometrium, with an adequate uterine blood supply until placental synthesis of progesterone begins. A detectable level of HCG begins at about 22 days from the LMP, which is approximately 8 to 11 days from conception. Initially, hcg levels rise exponentially, more than doubling each week during the first weeks in normal pregnancies. hcg levels over time during pregnancy. Once progesterone is produced by the placenta to maintain uterine function by stimulation of uterine growth and inhibits uterine contractions., hcg is no longer needed and the amount levels off and declines by approximately 12 to 14 weeks' gestation [8], in additional of pregnancy diagnosis hcg level is using to assessing fetal viability in threatened abortion, detecting ectopic pregnancy which fail to rise at expected rate.

Unconjugated estriol (uE3) chemistry is an estrogen with three-hydroxyl group produce in very large amounts during the last trimester of pregnancy; the biosynthetic pathway requires three organs to be fully functioning fetal adrenal, fetal liver and placenta. The fetal adrenal cortex possesses a unique zone for the production of steroids, the demand of estriol is so great that the fetal adrenal is massive compared with that of adult, the fetal adrenal avidly binds low-density lipoprotein to take into cholesterol, which is converted to tow major steroid intermediates pregnenolone sulfate and dehydroepiandrosterone sulfate (DHEAS), these intermediates are secreted into fetal circulation. The fetal liver possessing 16 $\alpha$-hydroxylase convert DHEAS to $16 \alpha-$ hydroxyl DHEAS, which is secreted, back to fetal circulation. Finally, the placenta synthesizes estriol from $16 \alpha-$ hydroxyl DHEAS. Approximately $90 \%$ of maternal serum estriol is derived from this pathway [37].

The clinical implication of maternal serum unconjugated estriol in addition to maternal serum screening using to assess function of fetoplacental unit, and decreasing of $\mathrm{uE} 3$ is association with risk of growth retardation, fetal death, down syndrome, fetal encephalopathy, fetus past maturity, preeclampsia, and Rh immunization [5].

Finally, technique which using in determination of three parameter in maternal serum for birth defect assessment by using Enzyme-Linked Immunosorbent Assay (ELISA) technique, for the quantitative in vitro diagnostic measurement of alpha fetoprotein, beta human chorionic gonadotropin and free estriol. 


\section{Materials and Methods}

Materials, a total of 91 pregnant ladies age range (20-45) years old in their $14^{\text {th }}$ to $20^{\text {th }}$ weeks of gestational age attending antenatal clinics in different hospitals of Khartoum state were included in the study. The hospitals were Asia Hospital, Almotakamla Health Center, China Friendship Hospital, Wad-nobawy Health Center, Alsalama Health Center, and Alshabia Health Center. Study subjects selected during a four months period starting from July to November 2007. The study is a prospective cohort where participants were followed up for an average period of 6.5 months until delivery. The sample population was divided into two groups; Study group including $69(75.8 \%)$ pregnant ladies with an age of $\geq 35$ years, and Control group including 22 $(24.2 \%)$ pregnant ladies with an age of $\leq 30$ years. History and back ground data were collected from participants using verbal interviews and pre-designed questionnaire (Appendix 1). Clinical and ultrasound examinations to determine the gestational age were performed by obstetricians and gynecologists. Five $\mathrm{ml}$ blood samples were collected from each participant; sera were separated, and then stored at $20^{\circ} \mathrm{C}$ for subsequent testing. Triple Screening Test was performed to all serum samples in Altoum-Sirageldin Medical Laboratory Centre. The test was performed to determine the concentrations of AFP, $\beta$-hcg, and Free estriol. Informed consents were obtained from all participants prior to involvement in the study. Ethical release to proceed in the study was obtained from the ethical committee of the Faculty of Medical Laboratory Sciences at Alneelain University. The study lasted for two years starting from March 2007 and ending in July 2009.

Methods, enzyme-Linked immunosorbent assay (ELISA technique) for the quantitative invitro diagnostic measurement of alpha-fetoprotein, beta human chorionic gonadotropin and free estriol in maternal serum.

Principle of the test, is a solid phase enzyme-linked immunosorbent assay, based on the sandwich principle. The microtiter wells wre coated with a monoclonal [mouse] antibody directed towards a unique antigenic site on an AFP and beta hcg molecules. An aliquot of patient sample containing endogenous molecules are incubated in the coated well with enzyme conjugate, which is an anti- AFP, and anti hcg antibodies conjugated with horseradish peroxidase. After incubation, the unbound conjugate was washed off. The amount of bound peroxidase is proportional to the concentration of AFP also hcg in the sample. Having added the substrate solution, the intensity of colour developed was proportional to the concentration of AFP, and hcg in the patient sample.

ELISA kit Competitive immunoenzymatic colorimetric method for quantitative determination of Free Estriol concentration in serum. Free Estriol (antigen) in the sample competes with horseradish-peroxidase Estriol (enzymeLabelle antigen) for binding onto the limited number of anti Estriol (antibody) sites on the microplates (solid phase). After incubation, the bound/free separation is performed by a simple solid-phase washing. The enzyme substrate $\left(\mathrm{H}_{2} \mathrm{O}_{2}\right)$ and the TMB-Sustrate (TMB) are added. After an appropriate time has elapsed for maximum colour development, the enzyme reaction is stopped and the absorbances were determined. Free Estriol concentration in the sample is calculated based on a series of standard. The colour intensity is inversely proportional to the Free Estriol concentration in the sample.

Calculations of Results, by using automated method, in this method the results have been calculated automatically using a 4 PL (4 Parameter Logistics) curve fit. 4 Parameter Logistics is the preferred method. The concentrations of the samples were read directly from this standard curve. Expected values during pregnancy shown in tables below.

Table 1. International expected values for AFP during pregnancy.

\begin{tabular}{llll}
\hline Weeks of pregnancy & $\begin{array}{l}\text { AFP } \\
{[\mathbf{I U} / \mathbf{m L}]}\end{array}$ & $\begin{array}{l}\text { Weeks of } \\
\text { Pregnancy }\end{array}$ & $\begin{array}{l}\text { AFP } \\
{[\mathbf{I U} / \mathbf{m L}]}\end{array}$ \\
\hline 10 & $9-24$ & 19 & $32-103$ \\
11 & $10-27$ & 20 & $42-121$ \\
12 & $10-30$ & 21 & $48-139$ \\
13 & $10-34$ & $22-24$ & $56-224$ \\
14 & $11-60$ & $25-27$ & $95-357$ \\
15 & $14-60$ & $28-30$ & $135-435$ \\
16 & $16-69$ & $31-33$ & $141-423$ \\
17 & $17-78$ & $34-36$ & $121-380$ \\
18 & $22-93$ & $37-40$ & $93-321$ \\
\hline
\end{tabular}

Table 2. International expected values for $\beta$-hcg during pregnancy.

\begin{tabular}{ll}
\hline $1^{\circ}$ week & $3.0-100 \mathrm{IU} / \mathrm{mL}$ \\
$2^{\circ}$ week & $10-1,000 \mathrm{IU} / \mathrm{mL}$ \\
$3^{\circ}$ week & $100-10,000 \mathrm{IU} / \mathrm{mL}$ \\
$4^{\circ}$ week & $1,000-100,000 \mathrm{IU} / \mathrm{mL}$ \\
$2^{\circ}$ month & $15,000-200,000 \mathrm{IU} / \mathrm{mL}$ \\
$3^{\circ}$ month & $10,000-100,000 \mathrm{IU} / \mathrm{mL}$ \\
$4^{\circ}$ month & $1000-10,000 \mathrm{IU} / \mathrm{mL}$ \\
\hline
\end{tabular}

Table 3. International expected values for free estriol during pregnancy.

\begin{tabular}{lll}
\hline Weeks & Median & Range IU/mL \\
\hline $14^{\circ}$ & 0.6 & $(0.2-3.0)$ \\
$15^{\circ}$ & 0.8 & $(0.2-3.5)$ \\
$16^{\circ}$ & 1.2 & $(0.3-4.2)$ \\
$17^{\circ}$ & 1.4 & $(0.4-5.2)$ \\
$18^{\circ}$ & 1.6 & $(0.4-5.8)$ \\
$19^{\circ}$ & 2.0 & $(0.4-6.2)$ \\
$20^{\circ}$ & 2.5 & $(0.4-6.8)$ \\
$22^{\circ}$ & 2.7 & $(0.4-9.1)$ \\
$24^{\circ}$ & 4.0 & $(0.4-9.1)$ \\
$26^{\circ}$ & 1.2 & $(1.9-9.5)$ \\
\hline
\end{tabular}

Quality control, the controls were running with each calibration curve for the three parameters, astatistically significant number of controls were assayed to establish mean values and acceptable ranges to assure proper performance. Control samples were used according to state and federal regulations. Using control sera at both normal and pathological levels.

The checking of the following technical areas: Pipetting and timing devices; photometer, expiration dates of reagents, storage and incubation conditions, aspiration and washing methods were done. 
Statistical analysis, Raw data were entered into a spread sheet of SPSS statistical package program, version 13. The data were rearranged as appropriate. Descriptive analysis was performed to all study variables. Data where tested for normality using Kilmogrove-Smirnove method. Chi-square test was performed to detect significant differences between study variables such as $\beta$-hcg level, AFP, and Free estriol with the pregnancy outcomes. Cross-tabulations were done as well to explore relations between study variables. To identify associations, their strength, and significance, Spearman's Correlation Coefficient was used.

\section{Results}

\subsection{Clinical Results}

Ninety one $(100 \%)$ pregnant ladies were enrolled in this study. They were distributed into two groups; Study group including $69(75.8 \%)$ pregnant ladies with an age of $\geq 35$ years, and Control group including $22(24.2 \%)$ pregnant ladies with an age of $\leq 30$ years.

All participants in the control group delivered normal healthy infants with a frequency of $10(45.5 \%)$ and 12 $(54.5 \%)$ male and female babies respectively. In the study group, participants delivered $57(82.6 \%)$ normal healthy infants with a frequency of $22(38.6 \%)$ and $35(61.4 \%)$ male and female babies respectively. Twelve ladies (17.4\%) delivered infants with congenital defects. The defects varied; four $(5.8 \%)$ had stillbirth, four $(5.8 \%)$ had abortion at the second trimester, three $(4.3 \%)$ had premature delivery, and one $(1.4 \%)$ had intrauterine death.

\subsection{Laboratory Results}

The present study showed differences of triple screening test results among study and control groups. Findings varied between normal, decreased, and increased in the levels of the three parameters. For instance, $\beta$-HCG levels detected among participants in the study group showed decreased level in $27.3 \%$, and normal level in $72.7 \%$ of samples. On the other hand, all participants in the control group had normal $\beta-\mathrm{HCG}$ level. AFP levels among the study group were normal in $82.1 \%$ of samples, decreased in $16.4 \%$, and increased in $1.5 \%$. In the control group, AFP levels were $81 \%$ which was considered as normal, and 19\% which was considered as decreased. Levels of free estriol among the study group were normal in $68.18 \%$ of samples, increased in $1.14 \%$, and decreased in $6.82 \%$. In the control group, all participants had normal unconjugated estriol levels.

Results of the Triple Screening Test showed variation in the concentrations of the three markers among both study and control groups. Mean concentration of $\beta-\mathrm{HCG}$ was $961.93 \mathrm{mlU} \backslash \mathrm{ml}$ in the study group, and $975.33 \mathrm{mlU} \backslash \mathrm{ml}$ in the control group. Mean concentrations of AFP were 25.70IU $\backslash \mathrm{ml}$ and $24.00 \mathrm{IU} \backslash \mathrm{ml}$ in the study and control groups respectively. Mean concentrations of free estriol were $0.77 \mathrm{ng} \backslash \mathrm{ml}$ and $0.86 \mathrm{ng} \backslash \mathrm{ml}$ in the study and control groups respectively. In the present study, the Triple Screening Test results obtained showed significant associations (P-value=0.023) with pregnancy age in both study and control groups. Chi-square test of significance was performed to all parameters of the Triple Screening Test against the pregnancy outcome in both study and control groups. Statistically significant differences between levels of $\beta$-HCG and pregnancy outcomes with a Pvalue of 0.000 were obtained using Pearson Chi-squire test of significance. Statistically insignificant differences between levels of AFP and pregnancy outcomes with a P-value of 0.5240 were detected. And highly significant differences between levels of free estiol and pregnancy outcomes were detected with a P-value of 0.000. Spearman's Correlation Coefficients were conducted between the study group and the abnormal pregnancy outcomes. Results showed significant $(\mathrm{P}$-value $=0.02)$ perfect correlation $(\mathrm{r}=1.000)$.

\section{Discussions}

Reaching to scientific evidence Through this cohort study that links congenital defects and abnormalities in newborns with being pregnant in an older age than 34 years. The rate of having a child with anomalies was statistically significant among women at age 35 years and above than among women at age 30 years and bellow.

Results indicating congenital defects obtained by the Triple Screening Test among Sudanese pregnant ladies were consistence with internationally known limits, indicating the usefulness of this test as screening for pregnancy abnormalities in Sudan. This finding goes hand in hand with what was obtained by the American Pregnancy Association presented in January 2008, and also with findings obtained by Temtamy et al among Egyptian pregnant ladies in 1998.

According to the national center for heath statistics of the United States of America, the risk of bearing a child with certain congenital disorders increases as a woman ages. For instance, at age 25 years, a woman has a 1-in-1250 chance of having a baby with birth defect. At age 30 years, the chance is 1-in-952. At age 35 years, 1 -in-378 chance. At age 40 years, 1-in-106 chance. And at 45 years, 1-in-30 chance. However, in this study, we found that the risk of having a baby with congenital defects is as high as $17.4 \%$ at an age of only 35 years and above!

The three markers of the Triple Screening Test yield results that fall within the normal range in the control group. In contrast, varying results were obtained in the study group. For instance, Beta-human Chorionic Gonadotropin ( $\beta-\mathrm{HCG})$ and free estriol (uE3) levels significantly decreased in all cases of abortion and still-birth. Alpha fetoprotein (AFP) level was also significantly decreased in all cases of abortion and still-birth as well as in all cases of premature delivery and intrauterine death. These findings were in complete agreement with results obtained by Yuan et al 2006.

Unfortunately, our study failed to Calculate the Multiples of the median (MoM) for Beta-human chorionic gonadotropin $(\beta-h c g)$, free estriol (uE3), and Alpha fetoprotein (AFP). This is justified by the fact that repeated blood samples should be collected every gestational week 
per each pregnant lady for the entire period of pregnancy (i.e. 40 weeks) to perform the Triple Screening Test. Participants refused to repeatedly expose to such invasive procedure. Also, Our limited financial resources were a major obstacle.

Although the Alpha fetoprotein (AFP) is a good predictor in birth defect screening tool in Europe and United States of America in an elderly pregnant ladies especially for Down syndrome screening but, in this study showed statistically insignificant differences between levels of AFP and pregnancy outcomes in an elderly Sudanese pregnant ladies.

\section{Conclusions}

By the end of this study, concluding that being pregnant in an older age is associated with having babies with congenital anomalies. Also demonstrate the possibility, applicability and, affecting of using simple, direct, and easy to perform procedures in the screening of important congenital defects in utero.

\section{References}

[1] Akbas SH, Zben TO, Alper O, et al., 2001. Department of biochemistry. Akdeniz University, Fucality of Midicine, Antalya, Turkey, Maternal serum screening for Down syndrome, open neural tube defects and trisome 18 .

[2] Allamn Gaw, Robert A Cowan, Denis St. J. O'Reilly, Michael J. Stewart, James Shepherd, Clinical biochemistry, second edition, 1999

[3] American Pregnancy Association, 2008. Pregnancy Symptoms-Early Signs of Pregnancy. APA. J. Anika R, Laura K, and Stanley K, 1998. A Global Review of Laws on Induced Abortion, International Family Planning Perspectives (Volume 24).

[4] Benn PA, Borgida A, Horne D, Briganti S, Collins R, and Rodis J, 1997. Down syndrome and neural tube defect screening: the value of using gestational age by ultrasonography.

[5] Bricker L, Garcia J, Henderson J, 2000. Ultrasound screening in pregnancy: Health technology assessment. Winchester, England.

[6] Chung C. S., Myrianthoupoulos N. C., 1975. Risks of Congenital Malformations. The National Foundation March of Dimes Original Article Series. (Volume 9), Page 10.

[7] Croen LA, Shaw GM, Sanbonmatsu L, and Selvin S, 1997. Maternal residential proximity to hazardous waste sites and risk for selected congenital malformations. Epidemiology 8 (4): $347-54$

[8] Davis F., 2005 Clinical Chemistry, A Laboratory Perspective Company.

[9] Deska Pagana, Kathleen, Pagana, and Timothy J. 2002. Mosby's manual of Diagnostic and Laboratory Tests, 2nd Edition.

[10] Donald I, MacVicar J, and Brown TG, 2005. Investigation of abdominal masses by pulsed ultrasound. PMID 13550965.1

\section{(7032): 1188-95}

[11] Edler I, Hertz CH, 2004. The use of ultrasonic reflectoscope for the continuous recording of movements of heart walls.

[12] Fombonne E, 2003. The prevalence of autism. JAMJ. 289: 8789.

[13] Goldman L, Apelberg B, Koduru S, Ward C, and Sorian R, 2001. Healthy From the Start. The Pew Environmental Health Commission.

[14] Haddow JE, Palomaki GE, Knight GJ, Cunningham GC, Lustig LS, and Boyd PA., 1994. Reducing the need for amniocentesis in women 35 years of age or older with serum markers for screening.

[15] Kasai C. 1985. Real-time two-dimensional blood flow imaging using an autocorrelation technique. IEEE Transactions on Sonics and Ultrasonics, 458-464.

[16] Krause, T. G. 2001. Second-trimester maternal serum alphafetoprotein and risk of adverse pregnancy outcome. Obstetrics \& Gynecology, volume 97, pages 277-282.

[17] Linda Nicholson, 2007. Birth defect assessments.

[18] Ljunger, E, Cnattingius, S, Lundin, C, \& Annerén, G. 2005 Chromosomal anomalies in first-trimester miscarriages. PMID 103628284 (11): 1103-1107.

[19] Loncar J, Barnabei VM, and Larsen JW, 1995 Advent of maternal serum markers for Down syndrome screening. Obstet Gynecol Surv; 50: 316-20.

[20] Marci JN, Kasturi RV, Krantz DA, CookEJ, Moore ND, Young JA, 1990. Maternal serum Down syndrome screening: free $\beta$-protein is amore effective marker than human chorionic gonadotropin. AM J Obstet Gynecol; 163: 1248-53.

[21] Moore K. \& Persaud T, 2003. The Developing Human: Clinically Oriented Embryology. W. B. Saunders Company.

[22] National Research Council. 2000. Scientific frontiers in developmental toxicology and risk assessment. National Academy Press; Washington DC.

[23] Natan Haratz-Rubinstein, 2006. Multiple screening markers.

[24] Kana S. A. 2003. Introduction to physics in modern medicine. Tsylor \& Francis. ISBN 0-415-30171-8.

[25] Nguyen, T. H, 1999. Evaluation of ultrasound-estimated date of delivery in 17450 spontaneous singleton births, Ultrasound in Obstetrics and Gynecology 14 (1): 23-28.

[26] Odutayo, Rotimi; 2007 Odunsi, Kunle Post Term Pregnancy.

[27] O'Rahilly and Muller F, 1987. Developmental stages in human embryos. Washington. Carnegie Institution of Washington.

[28] John Goldenring, 2004 Gestational age. Medline Plus Medical Encyclopedia: A. D. A. M., Inc.

[29] Smulian JC, Beres-Sochka L, DePrince K, Canterino J, Fischer R, Apuzzio J, Royle M. 2002 Birth defects surveillance. N J Med; 99 (12): 25-31.

[30] Steven Dowshen, 2007 MD Terrence U. H. Chun, November.

[31] Temtamy SA et al. 1998 A genetic epidemiological study of malformation at birth in Egypt. Eastern Mediterranean health journal, 4: 252-9. 
[32] Valerio D, Aiello R, and Fagnoni P, 2002. Genatic Research institute, Napoli.

[33] Wald NJ, Cuckle HS, Densem JW, Kennard A, and Smith D 1992. Maternal serum screening for Down's syndrome. Br J Obstet Gynaecol 99 No 2, 144-49.

[34] Wilcox AJ, Baird DD, and Weinberg CR, 1999. Time of implantation of the conceptus and loss of pregnancy. New Engl J Med. 340 (23): 1796-9.

[35] Yeargin-Allsopp M, Rice C, and Karapurkar T, 2003 Prevalence of autism in a US metropolitan area.
[36] CarlA. B urtis, Edward R. Ashwood, and David E. Bruns, 2005. TIETZ. Text book of clinical chemistry and molecular diagnostics fourth edition, (vol 1,2,4).

[37] Yuan-Yuan Wang, Jun Luo, 2006. a second trimester triple screening for Down syndrome. National Research institute for family planning, Beijing, China.

[38] Frances Fischbach, 1999. Manual of Laboratory and Diagnostic Tests. Sixth edition. 